\title{
Controlled trial of an ankle support (Malleotrain) in acute ankle injuries
}

\author{
J. O’Hara MB, ChB, J. C. Valle-Jones MRCS, LRCP*, H. Walsh MB, BS*, H. O'Hara MRCS, \\ N. B. Davey MB, BS, DA Engt, H. Hopkin-Richards MRCOG $\ddagger$ and R. M. Butcher BA, MB, \\ BChir§ \\ General Practitioners, Rottingdean, Sussex, *Burgess Hill, Sussex, †Shoreham, Sussex, $¥ B$ righton, Sussex and \\ §Godalming, Surrey, UK
}

\begin{abstract}
A randomized, controlled parallel-group trial has assessed 14 days' use of a new ankle support (Malleotrain, Bauerfeind, Aldershot, UK) in 220 patients (118 Malleotrain, 102 control group) with acute ankle injuries. Self-assessed pain levels were significantly lower in the group using Malleotrain at the end of the trial $(P<0.05)$, as were median times taken for reduction of symptom scores to $10 \%(P<0.05)$ and total analgesic consumption during the trial $(P<0.05)$. Overall clinical assessment scores were significantly superior in the Malleotrain group $(P<0.02)$. Of those patients who received Malleotrain, 112 of 116 patients who commented $(95 \%$ of all Malleotrain-treated patients) did so positively and only one patient stopped wearing the support during the trial. Malleotrain is acceptable to patients with acute ankle injuries and its use increases the rate of alleviation of symptoms. Its use should therefore be considered in the management of all such patients.
\end{abstract}

Keywords: Ankle injury, ankle support, orthotics

Ankle injuries are extremely common and may result from minor everyday trauma (e.g. stumbling and 'twisting' ankle) or from sporting injuries. In their mild and moderate forms, they represent the single most common type of minor trauma seen by general practitioners and accident departments.

The treatment of major ankle trauma involving bony injury or major ligamentous damage requires specialist attention, involving surgery and/or total immobilization of the affected joint. Treatment of the more common minor ankle injuries relies mainly on analgesia and various forms of supportive bandages or strapping of the affected joint. Frequent intensive physiotherapy can hasten recovery, but the logistics and cost of such treatment preclude it from being made available to the majority of patients. However, such injuries can result in a significant period of impaired mobility (sometimes preventing the patient from working) and/or sporting activity. Any form of readily available treatment which hastens recovery

Address for correspondence: Dr J. C. Valle-Jones, Consulting Centre, 11 Mill Road, Burgess Hill, Sussex, UK

Dr J. O'Hara died after completing revisions to the manuscript (C) 1992 Butterworth-Heinemann Ltd 0306-3674/92/030139-04 without burdening physiotherapy or other paramedical resources would therefore be valuable.

Malleotrain (Bauerfeind, Aldershot, UK) is a sophisticated elasticated ankle support with specially shaped silicone rubber inserts (Figure 1). These inserts not only maximize the support offered but also achieve constant massaging of the affected soft tissues, thereby emulating some of the techniques of physiotherapy which could be utilized. This aspect of treatment with Malleotrain has been described as 'active therapy'.

It has been suggested that the high quality of ankle support, together with the feature of 'active therapy' means that Malleotrain may speed resolution of symptoms and full recovery of ankle function ${ }^{1-3}$. If this can be confirmed, Malleotrain would represent a form of treatment superior to the standard therapy available to most patients, reducing the amount of

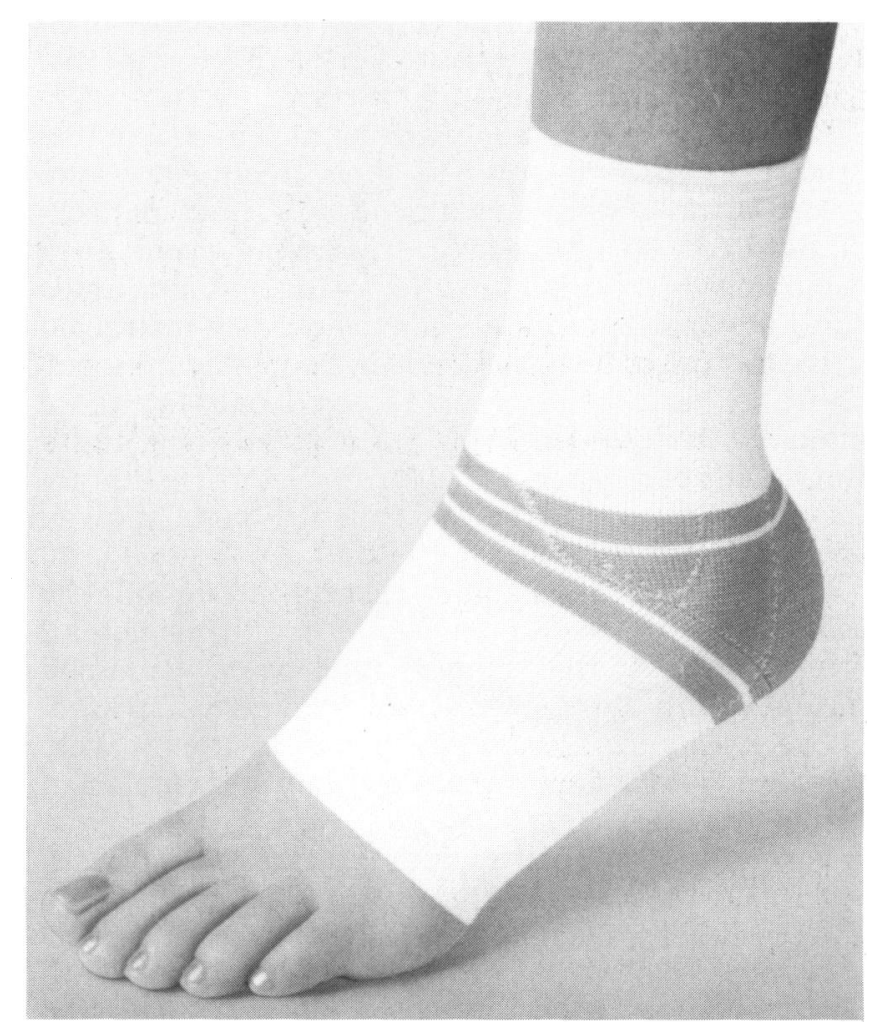

Figure 1. The Malleotrain ankle support 
time spent away from work and/or sporting activities and potentially reducing the number of medical and paramedical consultations required.

For these reasons, a controlled trial has been undertaken to evaluate the use of Malleotrain in the treatment of patients with acute ankle injuries.

\section{Patients and methods}

All patients who presented to the participating general practitioners with acute ankle injuries, unassociated with bony injury or major ligamentous damage, were considered for inclusion in the trial. Patients were acceptable for the trial if they were suffering their first ever ankle injury or an acute exacerbation of a longer standing problem, but not if they had only a chronic painful problem with the ankle (such as arthritis). Patients were also excluded from the trial if there was any suspicion of a bony injury or major ligamentous damage, if there was a history of ankle surgery or if they were receiving regular analgesics, non-steroidal anti-inflammatory agents or corticosteroids which could not be stopped for the period of the trial (simple analgesia on an 'as required' basis was allowed to continue throughout the trial). Patients whose size and/or shape was such that no standard size of Malleotrain would be suitable were also excluded.

Clinical examination and, where necessary, radiological examination were performed in order to confirm the absence of bony injury or major ligamentous injury.

Those patients fulfilling the entry criteria, and giving their informed consent to participate, were measured to determine what standard size of Malleotrain would be appropriate. If the patient's shape and/or leg measurement was such that none of the standard sizes of Malleotrain would have been suitable, the patient was excluded from the trial before the allocation of a trial number.

Patients were then allocated the next available trial number, which determined their treatment group according to a randomization code. Those patients randomized to the active treatment group then commenced treatment with a Malleotrain ankle support of the appropriate size. They were instructed to wear it every day, use while in bed being optional. Those in the control group received only 'standard therapy' advice on resting the affected joint and a simple support (such as Tubigrip, Seton Healthcare Group, Oldham, UK). All patients were allowed analgesia, if required.

Patients maintained daily records throughout the trial period and were reviewed by the investigator 2 weeks later. At that time patients were examined clinically and their overall improvement assessed. Those randomized to receive active treatment were questioned regarding the acceptability and comfort of Malleotrain.

Throughout the trial period, patients were permitted to take paracetamol (1g) up to four times per day, if necessary, for the relief of ankle pain. Consumption of paracetamol was recorded on the patients' 'diary cards' and was used as one of the indices of trial product efficacy. No other specific treatment for ankle pain was permitted but drugs for the treatment of unrelated disorders were allowed to continue.

\section{Assessments}

The primary assessments of treatment efficacy were on the basis of daily self-assessment by patients, using diary cards.

Pain self-assessment utilized $7-\mathrm{cm}$ horizontal Visual Analogue Scales (VAS), labelled 'none' at one end and 'worst imaginable' at the other. These scales were applied separately to pain in three situations at rest, during activities and during the night.

Limitation of activity was also assessed on a 7-cm VAS, labelled 'no limitation' at one end and 'no activity possible' at the other.

Patients also recorded whether they were able to work and the number of doses of analgesia taken during the day.

At the end of the trial, patients assessed their improvement on a 6 -point scale $(0$, much worse; 1 , slightly worse; 2 , no change; 3 , slightly better; 4, much better; 5 , cured).

At the end of the trial, patients were questioned regarding their current activity/work status. Those in the active treatment group were also questioned regarding the comfort and ease of use of Malleotrain.

\section{Statistical methods}

The significance of within-patient changes within groups was assessed using Wilcoxon's signed rank tests, Kolmogorov-Smirnov tests or paired $t$-tests. The two groups were compared using Mann-Whitney, Kolmogorov-Smirnov, $\chi^{2}$ and unpaired $t$-tests, as appropriate, applied to the withingroup changes during treatment.

In relation to the diary VAS data, two overall indices of symptoms were derived: areas under the response-time curves (trapezium method) and the time for each of the VAS scores to fall to $10 \%$ or less.

The number of patients in the trial was the number estimated to be required to achieve a power $(1-\beta)$ of 0.80 to detect a between-treatments difference of $20 \%$ in VAS responses at the $\alpha=0.05$ level. Two-tailed significance tests were used throughout and the threshold of significance was taken as $P=0.05$.

\section{Results}

\section{Patients}

A total of 220 patients (118 Malleotrain, 102 control) entered the trial - 153 men and 67 women of mean age 35.2 (range 14-78) years, mean weight 69.0 (range 44-101) kg and mean height 170.7 (range $155-188) \mathrm{cm}$. The two treatment groups were well matched as regards age, sex, weight and height.

In the majority of patients $(83 \%)$, symptoms were attributable to known recent minor trauma. The median (i.q.r.) duration of symptoms was 6 (3-12) days in the Malleotrain group and $5(2-11)$ days in the control group.

The two groups were well matched with respect to above-ankle measurements. In the group receiving 
Malleotrain, the sizes required were size 1 in $4 \%$ of patients, size 2 in $22 \%$, size 3 in $44 \%$, size 4 in $19 \%$ and size 5 in $11 \%$.

All patients completed the trial, returning for reassessment after 13-15 days (after reminder telephone calls when necessary).

\section{Visual analogue scores}

All VAS assessments showed similar patterns of results (Figures 2-5). Both groups showed progressive, highly significant (within-group), improvements during the 14 days of observation, in all cases the improvement being greater in the group on Malleotrain. In relation to all three modalities of pain (at rest, at night and during activity) and activity limitation, the differences between groups was statistically significant at the end of the trial $(P<0.05$; Student's $t$ test).

There was a consistent trend for lower 'areas under curves' (i.e. fewer symptoms) in the group receiving Malleotrain, although the difference was not statistically signficant (Student's $t$ test) in terms of pain at night or during activity (Table 1).

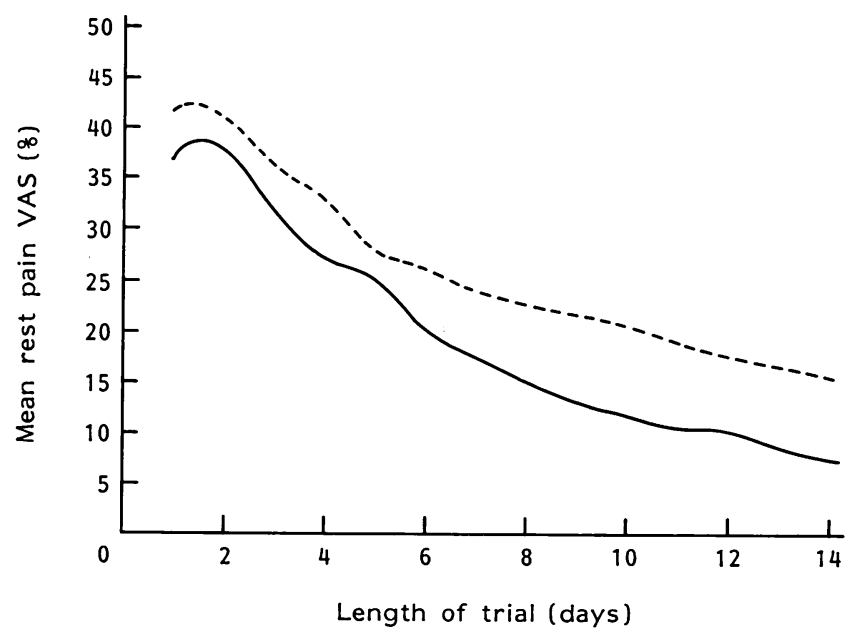

Figure 2. Rest pain VAS. At the end of the trial (Day 14), there was a significant difference between control (- - ) and treatment $(-)$ groups $(P<0.05 ;$ Student's $t$ test $)$

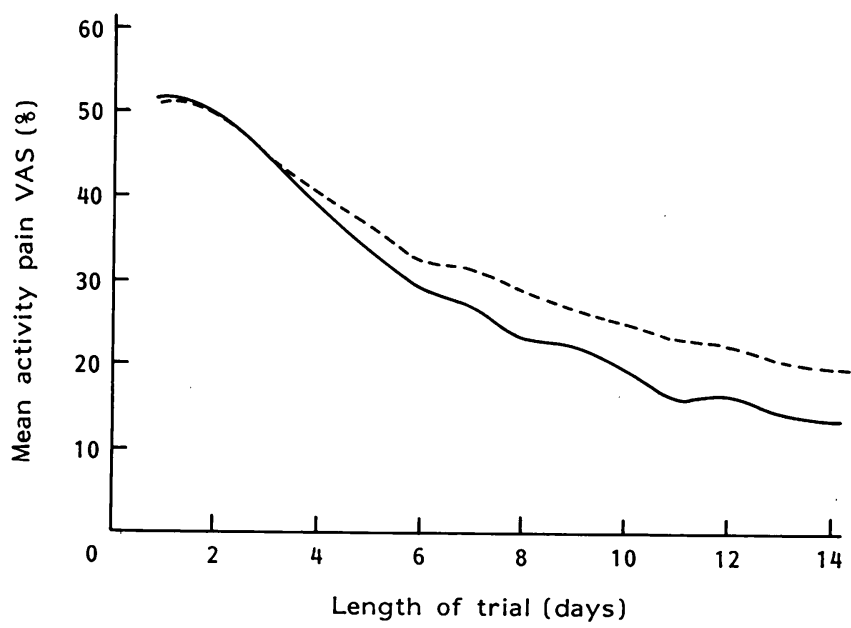

Figure 3. Activity pain VAS. At the end of the trial (Day 14), the difference between control $(---)$ and Malleotrain $(-)$ groups was significant $(P<0.05$; Student's $t$ test $)$

\section{Ability to work normally}

The number of patients able to work normally rose during the trial from $34 \%$ to $83 \%$ in the group on Malleotrain and from $36 \%$ to $81 \%$ in the control group - there was no significant difference $\left(\chi^{2}\right.$ test).

\section{Doses of analgesics}

Analgesic consumption fell progressively during the trial in both groups, and was consistently lower with

Table 1. Areas under VAS score-time curves

\begin{tabular}{lccc}
\hline Attribute & \multicolumn{2}{c}{ Mean area (units) } & $\begin{array}{c}\text { Significance } \\
\text { of difference } \\
\text { between groups }\end{array}$ \\
\cline { 2 - 3 } & $\begin{array}{c}\text { Malleotrain } \\
(n=118)\end{array}$ & $\begin{array}{c}\text { Control } \\
(n=102)\end{array}$ & \\
\hline Activity pain & 264.3 & 292.6 & n.s. \\
Rest pain & 178.5 & 235.8 & $<0.05$ \\
Night pain & 152.1 & 197.1 & n.s. \\
Activity limitation & 266.9 & 287.4 & n.s. \\
\hline
\end{tabular}

n.s. Not significant

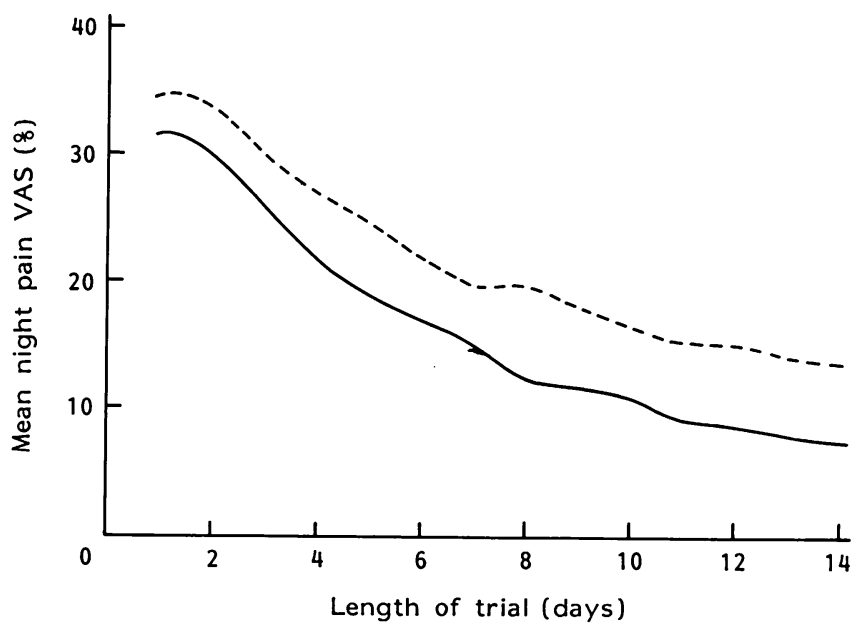

Figure 4. Night pain VAS. At the end of the trial (Day 14), the difference between control $(--)$ and Malleotrain $(-)$ groups was significant $(P<0.05 ;$ Student's $t$ test)

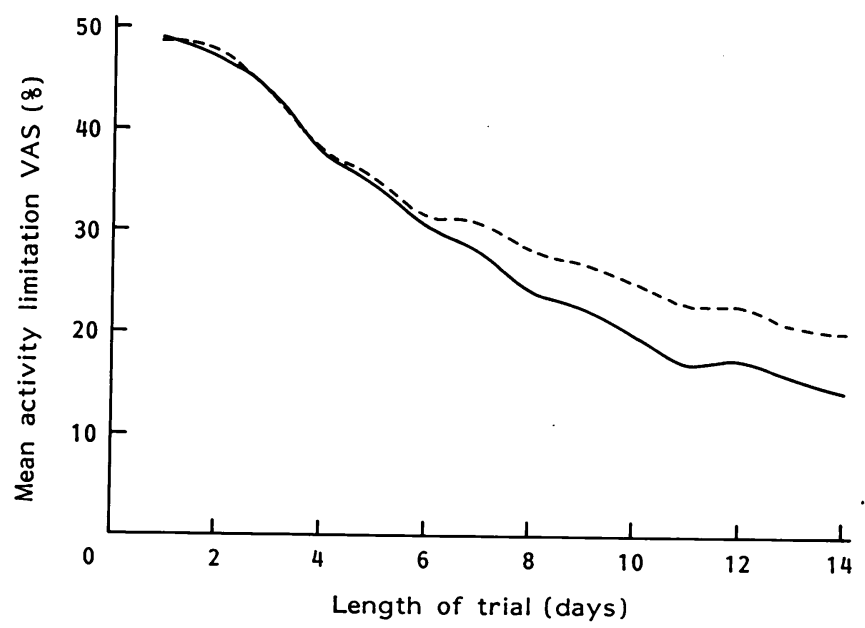

Figure 5. Activity limitation VAS. At the end of the trial (Day 14), the difference between control $(---)$ and Malleotrain $(\longrightarrow$ groups was significant $(P<0.05$; Student's $t$ test) 
Malleotrain. The median total number of doses of analgesia taken during the 14-day trial period was 11.0 in the Malleotrain group, compared with 25.6 in the control group $(P<0.05$; Mann-Whitney $U$ test).

\section{Speed of response}

A fall of VAS to $10 \%$ occurred more rapidly in the group on Malleotrain for all four assessments, significantly so in the case of pain at night and at rest; Mann-Whitney $U$ test. The improvement with Malleotrain was in the range 2-3 days (Table 2).

\section{Overall assessment}

The means of overall assessment scores were 4.37 units in the Malleotrain group and 3.94 units in the control group $(P<0.02 ;$ Kolmogorov-Smirnov test). Assessments of 'much better' or 'cured' (score 4 or 5) were seen in 104 of $118(88 \%)$ patients in the Malleotrain group, but only 69 of $102(68 \%)$ in the control group $\left(P<0.0005 ; \chi^{2}\right.$ test, Table 3$)$.

At the end of the trial, 92 of 105 Malleotrain-treated patients $(88 \%)$ were described as 'normal' or 'near normal', while this was only true of 63 of $94(67 \%)$ in the control group $\left(P<0.001 ; \chi^{2}\right.$ test) (no comment was made regarding the normality or otherwise of the remaining 13 patients in the Malleotrain group and the remaining 8 control patients).

Table 2. Time for VAS scores to fall to $10 \%$

\begin{tabular}{lccc}
\hline Attribute & \multicolumn{2}{c}{ Mean interval (days) } & \begin{tabular}{c} 
Significance \\
of difference \\
\cline { 2 - 3 }
\end{tabular} \\
\cline { 2 - 3 } $\begin{array}{c}\text { Malleotrain } \\
(n=118)\end{array}$ & $\begin{array}{c}\text { Control } \\
(n=102)\end{array}$ & \\
\hline Activity pain & 9.93 & 11.27 & n.s. \\
Rest pain & 9.13 & 11.38 & $<0.05$ \\
Night pain & 8.41 & 10.90 & $<0.05$ \\
Activity limitation & 10.26 & 11.54 & n.s. \\
\hline
\end{tabular}

n.s. Not significant

Table 3. Overall assessment of treatment

\begin{tabular}{|c|c|c|c|c|}
\hline Score & Assessment & $\begin{array}{l}\text { Malleotrain } \\
(n=118)\end{array}$ & $\begin{array}{c}\text { Control } \\
(n=102)\end{array}$ & $\begin{array}{c}\text { Significance } \\
\text { of difference } \\
\text { between } \\
\text { groups }\end{array}$ \\
\hline $\begin{array}{l}0 \\
1 \\
2 \\
3 \\
4 \\
5\end{array}$ & $\begin{array}{l}\text { Much worse } \\
\text { Slightly worse } \\
\text { No change } \\
\text { Slightly better } \\
\text { Much better } \\
\text { Cured }\end{array}$ & $\begin{array}{c}1(1) \\
1(1) \\
3(3) \\
9(8) \\
38(32) \\
66(56)\end{array}$ & $\begin{array}{l}0 \\
0 \\
10(10) \\
23(23) \\
32(31) \\
37(36)\end{array}$ & $\begin{array}{c}K=1.515 \\
P<0.02 \\
\text { (Kolmogorov- } \\
\text { Smirnov test) }\end{array}$ \\
\hline \multicolumn{2}{|c|}{ Mean score } & 4.37 & 3.94 & \\
\hline
\end{tabular}

Values in parentheses are percentages

\section{Comments on Malleotrain}

A total of 112 of the 116 (95\% of all Malleotraintreated patients) patients who commented on Malleotrain did so positively. Two patients considered the product 'heavy but good', one patient commented that it was initially uncomfortable but this problem resolved with continuing use, one said that the top edge cut into his leg and one patient stopped wearing the product (reason not stated). One patient disliked the colour. No one experienced any other problems with wearing Malleotrain.

\section{Discussion}

Two unavoidable difficulties arise in a clinical evaluation of a product such as Malleotrain - first, it is impossible to undertake a trial using the desirable rigorous 'blind' methodology; second, the 'placebo effect' could cause a problem, particularly in the study of disorders which are self-limiting. The resulting improvement in the 'control' group reduces the ability of the trial to distinguish between the active and control groups.

To minimize bias due to the non-blind methodology, a large trial was undertaken. The greatest emphasis has been placed on daily self-assessments by patients in their own homes, away from direct influence of the investigators. Patients were not faced with having to compare the two treatments but were merely reporting symptomatic changes in response to one or other of the treatments. There was thus less opportunity for systematic bias in the patients' self-assessments than might have been the case with investigator-involved assessments, since the investigator was inevitably assessing patients from both treatment groups. The trial design involved a large number of patients, estimated to give adequate statistical power.

However, despite the inevitable problems with a trial of this sort, the results demonstrate consistent differences between treatment groups. Malleotrain resulted in greater and more rapid improvement, significantly so in relation to the majority of assessment criteria. Overall assessments were significantly in favour of Malleotrain. Those patients who received the treatment nearly all commented favourably upon it - in only one case was its use discontinued.

This trial has demonstrated that, in patients with acute ankle injuries, a Malleotrain ankle support results in more rapid alleviation of symptoms than does Tubigrip and is acceptable to patients. Malleotrain should therefore be considered in the management of all patients with such injuries.

\section{References}

1 Hess $H$. Train-active supports for the management of sport injuries. Orthopädie Technik 1985; 7: 85

2 Hess $H$, Huberty $R$. Soccer injuries. In: Schneider RC, Kennedy JC, Plant ML, Fowler PJ, Hoff JT, Matthews LS, eds. Sports Injuries: Mechanisms, Prevention and Treatment. Baltimore, USA: Williams and Wilkins 1985, 163-177.

3 Hess $H$. Malleotrain-Bandage im klinischen Grossversuch Sportver Sportschaden 1991 (in press). 